\title{
Avaliação de percepção de fala em crianças com deficiência auditiva usuárias de aparelho de amplificação sonora: a questão do instrumento e seus critérios
}

\author{
Evaluation of speech perception in hearing impaired children: \\ the question of instrument and its criteria
}

\author{
Luana Almeida Magalhães ${ }^{1}$, Paula Martin Cimonari ${ }^{2}$, Beatriz Cavalcanti de Albuquerque Caiuby Novaes ${ }^{3}$
}

\begin{abstract}
RESUMO
Objetivo: Comparar diferentes critérios de análise de erros em tarefas de reconhecimento de palavras em crianças com deficiência auditiva usuárias de aparelho de amplificação sonora individual, visando estabelecer seu potencial de utilização na clínica, particularmente nas relações entre percepção de fala e utilização de audição residual. Métodos: Aplicação de listas para reconhecimento de palavras em sujeitos de 6 a 8 anos de idade, com deficiência auditiva e usuários de aparelho de amplificação sonora individual. As respostas foram analisadas por diferentes critérios a partir de dois instrumentos: WASP: acerto de palavras, de fonemas e padrão total de erro; Matriz de Confusão: substituições e omissões de fonemas. Resultados: As relações entre os diferentes critérios de análise permitiram diferentes perspectivas na análise do desempenho das crianças no reconhecimento de palavras, levando a reflexões sobre a utilização do potencial auditivo das crianças e as implicações no processo terapêutico com ênfase na audição residual. Conclusão: Combinar diferentes critérios de análise dos erros permite ampliar o conhecimento das habilidades de percepção de fala dos sujeitos e, conseqüentemente, contribui para o estabelecimento de metas terapêuticas.
\end{abstract}

Descritores: Deficiência auditiva; Percepção auditiva; Auxiliares de audição/utilização

\section{INTRODUÇÃO}

A intervenção fonoaudiológica em uma proposta oral para a criança com deficiência auditiva fundamenta-se na utilização da audição residual. Para tanto, atualmente, a Fonoaudiologia conta com inúmeros instrumentos de avaliação sistematizados de percepção auditiva, que podem ser de grande valia em pesquisas nesta área de conhecimento. Um trabalho fonoaudiológico, voltado para o uso da audição residual com o auxílio de aparelho de amplificação sonora individual (AASI), permite a criança surda receber a maior

(1) Pós-graduanda do Programa de Estudos Pós-Graduados em Fonoaudiologia da Pontifícia Universidade Católica de São Paulo - PUC-SP - São Paulo (SP), Brasil.

(2) Bolsista de iniciação científica do Conselho de Ensino e Pesquisa da PUCSP - CEPE 2002.

(3) Doutora, Professora Titular da Pontifícia Universidade Católica de São Paulo - PUC-SP - São Paulo (SP), Brasil.

Trabalho realizado na Pontifícia Universidade Católica de São Paulo - PUCSP - São Paulo (SP), Brasil e na Divisão de Educação e Reabilitação dos Distúrbios da Comunicação da Pontifícia Universidade Católica de São Paulo - DERDIC - São Paulo (SP), Brasil.

Endereço para correspondência: Beatriz C. A. Caiuby Novaes. Al. Tietê, 111/91, São Paulo - SP, CEP 01417-020. E-mail: beatriznovaes@ pucsp.br

Recebido em: 8/11/2006; Aceito em: 20/8/2007 quantidade possível de informação acústica dos sons da língua $^{(1-2)}$. Quanto melhor a criança surda puder utilizar-se das informações acústicas, maiores chances para o desenvolvimento da linguagem oral ela terá(3-4).

Propostas desta natureza buscam oferecer à criança as melhores oportunidades para desenvolver suas habilidades linguiísticas da forma mais completa possível, para que possa vir a ser lingüisticamente competente, pelo menos na língua de sua família ${ }^{(5-8)}$. Nesta tendência, tratar do surdo para integrá-lo à sociedade passou a ser uma postura que não só permeou a área da surdez como passou a ser parte da proposta de reabilitação e/ou educação especial relativa a sujeitos com alguma deficiência. Nos dias de hoje, este conceito está atrelado à inclusão social ${ }^{(9)}$, em uma condição democrática de integração do sujeito com deficiência à sociedade, garantindo-lhe as mesmas oportunidades que as dadas aos demais para o desenvolvimento de suas potencialidades, por meio do convívio, respeito e cooperação da sociedade ${ }^{(10)}$.

A percepção dos sons da fala inclui a recepção e interpretação dos padrões de fala; a discriminação entre os sons de diferentes espectros, durações, características temporais, formas seqüenciais e ritmo; o reconhecimento, a memorização e a compreensão de unidades de fala dentro de determinado sistema lingüístico ${ }^{(11)}$. Os efeitos de uma perda audi- 
tiva neurossensorial na percepção de fala constituem-se na redução da habilidade de identificar contrastes fonológicos significantes, de reconhecer fonemas e de realizar a figurafundo auditiva. Crianças com perda auditiva periférica podem ter dificuldades quantitativas e qualitativas para reconhecer as pistas acústicas da fala, que podem resultar em alterações da produção da fala ${ }^{(12-13)}$.

A avaliação da percepção de fala através de AASI tem sido bastante estudada ${ }^{(14-20)}$. A maioria destes estudos buscou caracterizar a percepção de fala de adultos e crianças deficientes auditivos. No entanto, estudos que considerem a relação percepção e produção de fala e, conseqüentemente, a relação entre o processo de ajuste na amplificação e a percepção de fala ainda se fazem necessários. A inteligibilidade, a variabilidade dos sujeitos e a dificuldade de aplicação de provas com crianças têm sido vistos como problemas na realização de pesquisas desta natureza ${ }^{(21-22)}$.

A utilização de matrizes de confusão foi amplamente estudada e discutida ${ }^{(23)}$, sendo que diversos estudos na Lingüística demonstraram seu potencial na indicação de dispositivos de amplificação e análise de condições de percepção de deficientes auditivos. Na Fonoaudiologia foi recentemente proposta por alguns autores ${ }^{(20,24)}$ cujo principal interesse tem sido compreender o potencial da matriz de confusão de fonemas no sentido de apreciar o tipo, a natureza e quantidade dos erros apresentados nas consoantes que melhor caracterize o potencial de percepção auditiva dos sujeitos para os sons da fala.

Outra proposta de análise qualitativa de erros em tarefas de reconhecimento de palavras é a Associação de Palavras para Percepção de Sílabas, desenvolvido por autoras ${ }^{(25)}$ americanas como parte de um Programa de Percepção e Produção de Fonemas, nomeado como Word Associations for Syllable Perception (WASP, cuja sigla será utilizada neste artigo a partir deste momento). O potencial deste instrumento na adapatção de AASI e no acompanhamento terapêutico de crianças com deficiência auditiva foi investigado ${ }^{(26)}$, tendo em vista que ouvir por meio de um dispositivo de amplificação gera dificuldades específicas para a criança deficiente auditiva e, consequentemente, leva à dificuldades na aquisição da linguagem oral. Assim sendo, a autora sistematiza, na língua portuguesa, atividades a serem realizadas semanalmente e registradas com precisão no sentido de promover o que denominda "reconhecimento instantâneo do fonema" (26).

No presente estudo, o objetivo foi comparar diferentes critérios de análise de erros em tarefas de reconhecimento de palavras em crianças com deficiência auditiva usuárias de aparelho de amplificação sonora individual, visando estabelecer seu potencial de utilização na clínica, particularmente nas relações entre percepção de fala e utilização de audição residual. Este é um estudo clínico e, nesta medida, privilegia a singularidade de cada sujeito e busca ao mesmo tempo analisar tendências e semelhanças que contribuam para a construção de conhecimento na Fonoaudiologia. Desta forma, foram analisadas as vantagens e desvantagens dos diferentes critérios de análise das respostas no reconhecimento de palavras.

\section{MÉTODOS}

Esta pesquisa foi aprovada pelo Comitê de Ética em Pesquisa do CEPE/PUC-SP sob o número do protocolo 0143/ 2003. Para a seleção dos sujeitos, foram determinados critérios que correspondessem com os objetivos da pesquisa, quais sejam: crianças deficientes auditivas, sem comprometimentos neurológicos ou de sistema orofacial, com faixa etária entre seis e oito anos e em atendimento clínico fonoaudiológico, tendo a modalidade oral como principal modo de comunicação, a partir da qual houvesse repertório de vocabulário para aplicação de lista de palavras.

Foram selecionadas quatro crianças com deficiência auditiva, três do sexo feminino e uma do sexo masculino, com idades entre seis e oito anos, tendo sido identificados por: $\mathrm{Ka}, \mathrm{An}$, Gui e Re. Todas as crianças eram atendidas no Programa de Audiologia Educacional da clínica-escola da DERDIC - Divisão de Educação e Reabilitação dos Distúrbios da Comunicação (PUC-SP), apresentavam perda auditiva neurossensorial bilateral, sendo todas usuárias de aparelho de amplificação sonora individual. Uma vez que se trata de um estudo que acompanha as características de cada sujeito, apresentamos a média das freqüências de 500, $1 \mathrm{~K}, 2$ $\mathrm{K}$ e $4 \mathrm{KHz}$ no melhor ouvido de cada criança, qual seja: Ka: 77,5 dB; An: 73,7 dB; Gui: 40 dB; Re: 80 dB. Observa-se que uma criança, Gui, diferencia-se no grau de perda auditiva quando comparada com as outras crianças avaliadas no estudo. No entanto, por se tratar de uma análise intra-sujeito, as diferenças observadas são incluídas na análise que será demonstrada posteriormente.

Para estes sujeitos a linguagem oral era a principal modalidade de comunicação, inclusive nos atendimentos que aconteciam há, no mínimo, seis meses. Além disso, não apresentavam comprometimentos do sistema sensório motor oral que pudessem interferir em suas produções de fala. Os seus responsáveis assinaram termo de consentimento informado contendo o objetivo e o procedimento da pesquisa, para que esta fosse devidamente permitida.

\section{Caracterização dos sujeitos}

Caso $\mathrm{Ka}$ : criança do sexo feminino, com sete anos de idade e em atendimento fonoaudiológico há dois anos, na época da pesquisa. Apresentava perda auditiva neurossensorial bilateral com média nas frequiências de 500, 1 K, 2 $\mathrm{K}$ e $4 \mathrm{KHz}$ de 77,5 dB no ouvido melhor, utilizando AASI, sendo que a seleção, adaptação, indicação e verificação das características eletroacústicas foram garantidas antes da avaliação no momento da pesquisa. Ka freqüentava a pré-escola de educação infantil, cuja abordagem não utilizava língua de sinais. Com relação à terapia fonoaudiológica, não se observava alteração na produção de fala, ou seja, apresentava fala inteligível, de acordo com a avaliação da terapeuta. Desta forma, a ênfase do trabalho era fundamentada na utilização da audição residual, visando a linguagem verbal oral.

Caso An: criança do sexo masculino, com seis anos de idade na época da pesquisa. Há dois anos do início da pesquisa, procurou a instituição para indicação de AASI e tera- 
pia fonoaudiológica. Apresentava perda auditiva neurossensorial bilateral com média nas freqüências de $500,1 \mathrm{~K}, 2 \mathrm{~K}$ e $4 \mathrm{KHz}$ de 73,7 dB no ouvido melhor, utilizando AASI, sendo que a seleção, adaptação, indicação e verificação das características eletroacústicas foram garantidas antes da avaliação no momento da pesquisa. An freqüentava escola de educação infantil, cuja abordagem não utilizava a língua de sinais. Com relação à terapia fonoaudiológica, An foi atendido na mesma instituição e sua fala apresentava-se inteligível, de acordo com a avaliação da terapeuta.

Caso Gui: criança do sexo masculino, com oito anos de idade na época da pesquisa. Um ano antes do início da pesquisa, procurou a instituição para indicação de AASI. Apresentava perda auditiva neurossensorial bilateral com média nas freqüiências de 500, 1 K, $2 \mathrm{~K}$ e $4 \mathrm{KHz}$ de 40 dB no ouvido melhor, utilizando AASI, sendo que a seleção, adaptação, indicação e verificação das características eletroacústicas foram garantidas antes da avaliação no momento da pesquisa. Após a aquisição do AASI, Gui foi encaminhado ao Posto de Saúde próximo de sua residência, para receber atendimento fonoaudiológico e, neste local, ocorreu a aplicação das listas de palavras por uma das pesquisadoras, que não observou alteração na sua produção de fala, ou seja, apresentava fala inteligível. Ele freqüentava a $2^{\mathrm{a}}$ série do Ensino Fundamental e não utilizava a língua de sinais. Vale ressaltar que o diagnóstico da deficiência auditiva somente foi realizado quando Gui tinha seis anos, o que levou os médicos a pensarem em uma perda auditiva progressiva. Desta forma, realizava monitoramento auditivo periodicamente (de seis em seis meses) para acompanhar a evolução da perda auditiva.

Caso Re: criança do sexo masculino, com sete anos de idade na época da pesquisa. Há um ano do início da realização da pesquisa procurou a instituição para realizar processo de indicação de AASI. Apresentava perda auditiva neurossensorial bilateral com média nas frequiências de 500, $1 \mathrm{~K}, 2 \mathrm{~K}$ e $4 \mathrm{KHz}$ de $80 \mathrm{~dB}$ no ouvido melhor, utilizando AASI, sendo que a seleção, adaptação, indicação e verificação das características eletroacústicas foram garantidas antes da avaliação no momento da pesquisa. Após a aquisição do AASI, Re foi encaminhado ao Posto de Saúde próximo de sua residência, para receber atendimento fonoaudiológico e, neste local, ocorreu a aplicação das listas de palavras por uma das pesquisadoras, que não observou alteração na produção de fala, ou seja, ele apresentava fala inteligível. Re frequientava $1^{\text {a }}$ série do Ensino Fundamental e sua escola utilizava a língua de sinais em sua proposta pedagógica.

\section{Material}

\section{WASP}

Seguindo o modelo proposto pelas autoras ${ }^{(25-26)}$, foram elaboradas seis listas de palavras pelas pesquisadoras. As listas apresentam diferentes graus de dificuldades e foram modificadas em função da criança a ser avaliada, uma vez que todos os vocábulos das listas deviam fazer parte do repertório linguiístico da mesma, de modo que as palavras utilizadas fossem escolhidas em função da familiaridade que apresentavam no vocabulário da criança (Anexo 1).
Durante o procedimento de avaliação, duas fonoaudiólogas, responsáveis pela pesquisa, apresentaram as palavras das listas às crianças, que deveriam repetir o que foi dito da forma que entenderam. Optou-se pelo procedimento de vivavoz, com treinamento da velocidade de fala e da intensidade vocal pelas profissionais, além de controle da distância entre terapeuta, anteparo e criança, o que tornou o possível a apresentação do estímulo durante o período de atenção da criança e, da mesma forma, preservar a familiaridade da criança com o ambiente, tendo em vista que se tratavam de crianças que se encontravam em atendimento terapêutico. A tarefa era realizada de modo que a criança repetisse as palavras sem alternativas de respostas e sem possibilidade de repetição durante a aplicação das listas de palavras.

Para tanto, um anteparo foi posicionado na frente da avaliadora, a fim de evitar o uso de pista visual por parte da criança. $\mathrm{O}$ anteparo utilizado foi feito de tecido para alto falante, visando não alterar as características acústicas do estímulo ${ }^{(25)}$. A sala utilizada para realizar a avaliação dos sujeitos era bem iluminada e uma criança e a avaliadora posicionaram-se frente a frente, a uma distância de aproximadamente um metro.

Seguindo o critério de familiaridade ao vocabulário, nem sempre todas as listas foram aplicadas em todos os sujeitos. Algumas das crianças apenas foram submetidas a duas listas (Re) e outras a quatro (An, Gui) ou cinco listas (Ka) e essa diferença decorreu do pouco repertório oral de algumas crianças pela faixa etária e/ou momentos do processo de aquisição de linguagem. A variação entre a quantidade de listas aplicadas se deu pela familiaridade das palavras apresentadas pelas crianças. Desta forma, a aplicação de uma ou mais listas para cada criança não interfere no número total de palavras, já que representa o número total de fonemas apresentados, ou seja, o número de estímulos ao qual a criança foi submetida. Desta forma, o número de estímulos depende da contagem fonêmica e de palavras, portanto, não interferem nos resultados da pesquisa a criança ter sido submetida a mais listas de palavras do que outras.

Cada lista era formada por 24 vocábulos, buscando a ocorrência dos fonemas na língua portuguesa brasileira, mas sem a intenção de realizar balanceamento fonético, já que o critério principal para a escolha dos vocábulos era a familiaridade sendo, portanto, as palavras mais facilmente produzidas pelas crianças. $\mathrm{O}$ fato da lista de palavras ser ou não ser foneticamente balanceada não influencia nos valores de reconhecimento das palavras em pacientes com perdas neurossensoriais ${ }^{(27)}$. Desta forma, o uso das listas deve privilegiar o repertório de palavras das crianças e, por isso, devem ser criadas pelos fonoaudiólogos que as utilizarem.

Obteve-se então, um total de 144 palavras, 652 fonemas, 320 vogais e 322 consoantes, sendo que as seguintes palavras, que constavam em cada lista, eram agrupadas segundo suas características:

Lista 1: dó, dia, eu, boi, lua, tia, rua, uva, pá, pé, pão, pai, mãe, não, meu, oi, nó, ovo, lá, ir, sol, céu, mão, seu;

Lista 2: vaca, casa, lixo, bola, dado, gato, pato, tatu, pipa, faca, cama, chave, sapo, rato, mala, lobo, gelo, cola, boca, novo, pano, fogo, doce, foto; 
Lista 3: dor, giz, paz, luz, par, som, mar, bar, ver, bis, gol, mulher, homem, colar, bolas, caçar, chover, zíper, varal, colher, chorar, pular, patas, papel;

Lista 4: time, pilha, data, fofo, tiro, táxi, moça, fora, pêra, cura, palha, sofá, café, babá, sino, você, manhã, purê, bode, mamá, vovó, maçã, nenê, cajú;

Lista 5: tubarão, jacaré, picolé, macarrão, caminhão, dominó, camarão, buzina, estojo, polícia, bigode, relógio, pedágio, garota, rápido, música, mágico, árvore, ônibus, pássaro, cócega, número, xícara, bêbado;

Lista 6: galinha, cavalo, bolacha, sapato, macaco, barata, piscina, carrinho, boneca, novela, panela, menina, pijama, bexiga, dinheiro, girafa, comida, caneta, velhinho, ratinho, desenho, palhaço, tesoura, família.

Na lista 3, a produção do arquifonema nos verbos no infinitivo não foi considerada erro, pois ocorre comumente na linguagem oral cotidiana, devido a variações lingüísticas regionais ${ }^{(20)}$.

\section{Matriz de Confusão}

Após a aplicação do protocolo WASP, realizou-se uma análise da discriminação e do reconhecimento de palavras pelas crianças por meio da Matriz de Confusão(20,23), a qual permite uma análise mais detalhada das características dos acertos, das omissões e substituições dos fonemas ${ }^{(20)}$. A Matriz de Confusão elaborada pela autora contém somente os fonemas consonantais falados no português brasileiro: $/ \mathrm{p} /, / \mathrm{t} /$, /k/, /b/, /d/, /g/, /f/, /s/, /s/, /t J/, /v/, /z/, /3/, /dz/, /m/, /n/, /n/, $/ \mathrm{l} / \mathrm{\lambda} / \mathrm{,} / \mathrm{r} / \mathrm{e} / \mathrm{R} /$.

Os dados inseridos na Matriz de Confusão resultam na comparação de 21 fonemas consonantais, visualizada no cruzamento desses fonemas, o qual representa os resultados de fonemas apresentados como estímulo (E) versus fonemas emitidos como resposta $(\mathrm{R})$, anotados em cada célula da Matriz de Confusão.

Em cada célula da matriz de confusão encontram-se os pares de respostas possíveis, sendo que o valor encontrado dentro das células demonstra a frequiência de ocorrência de resultado obtido para cada fonema. As respostas traduzem o acerto ou a substituição do fonema emitido, a diagonal principal, destacada em cinza claro, traduz a emissão correta do fonema e a quantidade de substituições é observada pelo número encontrado na célula de cruzamento, já os sub-grupos, ao redor da diagonal principal, destacam os traços lingüísticos por modo de articulação. As emissões dos pacientes caracterizaram-se em acertos, substituições e omissões dos fonemas apresentados durante a avaliação da percepção/ produção de fala ${ }^{(20)}$.

\section{Procedimentos}

\section{WASP adaptado}

\section{Registro das Respostas:}

Nas listas, foram utilizados os seguintes parâmetros para caracterizar as consoantes, no que se refere aos seus traços lingüísticos. Na descrição fonética do português são utilizados os seguintes parâmetros: Modo de articulação $(M)$ : tipo de obstrução no trato vocal durante a produção da consoante; Ponto de articulação $(P)$ : região da produção no trato vocal e os órgãos que participam da produção da consoante e Vozeamento ( $V)$ : ausência ou presença de vibrações das cordas vocais na passagem do ar pela laringe na produção da consoante ${ }^{(26)}$.

A forma de avaliação do teste é baseada nas respostas das crianças. Na folha de respostas, os espaços em branco foram preenchidos com símbolos, de acordo com a produção do paciente ${ }^{(26)}$ : quando era produzido o fonema correto como resposta ao estímulo sonoro, marcava-se $\sqrt{ }$; se ele omitisse o fonema apresentado, marcava-se $\varnothing$ e se ele dissesse outro fonema que não o apresentado, ou seja, se errasse, o espaço ficava em branco ([ ]). Se o fonema apresentado não ocupasse todos os espaços para preenchimento das características acústicas da lista, o espaço não ocupado pelo fonema apresentado era marcado com [- $]$ para evidenciar que não houve falha do paciente durante a repetição, mas sim que não havia o que ser preenchido no determinado espaço. As percentagens foram calculadas em relação ao número total de palavras na lista.

A seguir ilustramos exemplos de anotações nas listas: no Quadro 1, observa-se que na antepenúltima coluna da direita são anotadas as omissões dos respectivos fonemas. A última coluna da direita indica o número total de apresentações de cada fonema na lista de vocábulos que foi utilizada durante a avaliação. Já no Quadro 2, observa-se exemplo de lista de

Quadro 1. Exemplo de registro de palavra apresentada, resposta do sujeito, marcação segundo traços de modo, vozeamento e ponto articulatório das consoantes, além do acerto das vogais e pontuação total da palavra.

\begin{tabular}{|c|c|c|c|c|c|c|c|c|c|c|}
\hline \multirow[t]{2}{*}{ Palavra } & \multirow[t]{2}{*}{ Aproximação } & \multicolumn{3}{|c|}{ Consoante inicial } & \multirow[t]{2}{*}{ Vog } & \multicolumn{3}{|c|}{ Consoante final } & \multirow[t]{2}{*}{ Vog } & \multirow[t]{2}{*}{ Total } \\
\hline & & M & V & $P$ & & M & V & $\mathrm{P}$ & & \\
\hline Pato & Bato & $\sqrt{ }$ & & $\sqrt{ }$ & $\sqrt{ }$ & $\sqrt{ }$ & $\sqrt{ }$ & $\sqrt{ }$ & $\sqrt{ }$ & $7 / 8$ \\
\hline
\end{tabular}

Quadro 2. Exemplo de registro de palavra apresentada, resposta do sujeito, marcação segundo traços de modo, vozeamento e ponto articulatório das consoantes, além do acerto das vogais e pontuação total da palavra.

\begin{tabular}{|c|c|c|c|c|c|c|c|c|c|}
\hline \multirow[t]{2}{*}{ Palavra } & \multirow[t]{2}{*}{ Aproximação } & \multicolumn{3}{|c|}{ Consoante inicial } & \multirow[t]{2}{*}{ Vog } & \multicolumn{3}{|c|}{ Consoante final } & \multirow[t]{2}{*}{ Total } \\
\hline & & M & V & $\mathrm{P}$ & & $\mathrm{M}$ & V & $\mathrm{P}$ & \\
\hline Luz & Lú & $\sqrt{ }$ & $\sqrt{ }$ & $\sqrt{ }$ & $\sqrt{ }$ & & & & $4 / 7$ \\
\hline
\end{tabular}


palavras cuja característica é indicada para crianças que não tem condições de produção de palavras dissilábicas ou trissilábicas. No caso de crianças com repertório lingüístico restrito, é dada a possibilidade de criação de lista de onomatopéias, para testar a percepção auditiva.

As produções das crianças foram transcritas e analisadas segundo diferentes critérios: a) percentagem de acerto de palavras, b) percentagem de acerto de fonemas, c) padrão total de erro de fonemas, d) padrão total de erros para vogais $^{(25)}$, e) padrão total de erro para consoantes (adaptado pelas autoras), e preenchimento da matriz de confusão, incluindo f) percentagem de acerto de consoantes, g) substituição de consoantes e h) omissões de consoantes. O desempenho dos sujeitos foi medido pelo número correto de palavras repetidas e as percentagens foram calculadas em relação ao número total de palavras apresentadas.

\section{Matriz de Confusão}

\section{Registro das Respostas:}

$\mathrm{Na}$ Matriz de Confusão elaborada pela autora(20), os fonemas consonantais que se encontram na primeira coluna da esquerda representam os fonemas/estímulos, enquanto os fonemas encontrados na horizontal superior representam, os fonemas/respostas. Na antepenúltima coluna da direita, preenchida em amarelo claro, são anotadas as omissões dos respectivos fonemas. A última coluna da direita indica o número total de apresentações de cada forma na lista de vocábulos que foi utilizada durante a avaliação. Na linha superior-esquerda para a linha inferior-direita, os destaques são caracterizados pelos sub-grupos de distinção entre os fonemas plosivos surdos, plosivos sonoros, fricativos e africados surdos, fricativos e africados sonoros, nasais, líquidos laterais e líquidos não laterais.

As mesmas respostas (emissões dos pacientes) foram transpostas para as matrizes de confusão, considerando-se somente as consoantes. Os resultados foram caracterizados na matriz de confusão por percentagem de acerto de consoantes, percentagem de erro (ocorrência de substituições fonêmicas) e percentagem de omissões das consoantes. Optou-se por utilizar a Matriz de Confusão, pois esta possibilita ao avaliador uma análise qualitativa do padrão de erro, uma visualização do tipo, da natureza e da quantidade de erros apresentados ${ }^{(28)}$.

\section{Análise dos dados}

O desempenho das quatro crianças na tarefa de reconhecimento de palavras foi analisado através de cinco critérios diferentes para o WASP e três critérios para a Matriz de Confusão.

\section{WASP}

a) percentagem de palavras corretas:

$\frac{\text { número de palavras repetidas corretamente }}{\text { número total de palavras apresentadas }}$ X 100

b) percentagem de fonemas corretos:

$\frac{\text { número de fonemas repetidos corretamente }}{\text { número total de fonemas das palavras }}$ X 100

c)percentagem de traços corretos (consoantes e vogais)

número de traços reconhecidos corretamente número total de traços passíveis de uma palavra X 100

d) percentagem de traços corretos (consoantes):

número de traços reconhecidos corretamente nas consoantes

número de consoantes apresentadas

X 100

e) percentagem de traços corretos (vogais)

número de traços reconhecidos corretamente nas vogais

número de vogais apresentadas X 100

\section{Matriz de Confusão}

f) percentagem de fonemas consonantais repetidos corretamente

g) percentagem de fonemas consonantais substituídos por outro

h) percentagem de fonemas consonantais omitidos

Estes dados serão analisados conjuntamente no contexto de cada caso clínico apresentado.

\section{RESULTADOS}

\section{Caso Ka}

O desempenho de Ka está descrito na Tabela 1 e observase que, na aplicação da lista de palavra, foram verificados $27 \%$ de acerto de palavras, $68 \%$ de acerto de fonemas, $70 \%$ de padrão total de erros de fonemas, $56 \%$ de padrão total de erro de consoantes e $85 \%$ de padrão total de erro de vogais. À Matriz de Confusão (Anexo 2), houve $48 \%$ de acerto de consoantes, $41 \%$ de substituições e $11 \%$ de omissões de consoantes.

Tabela 1. Descrição do desempenho de Ka na tarefa de reconhecimento de palavras

\begin{tabular}{lccc}
\hline & N & Total & $\%$ \\
\hline WASP & 32 & 120 & $27 \%$ \\
Acerto de palavras & 332 & 490 & $68 \%$ \\
Acerto de fonemas & 690 & 979 & $70 \%$ \\
Padrão total de erro de fonemas & 732 & $56 \%$ \\
Padrão total de erro para consoantes & 413 & 247 & $85 \%$ \\
Padrão total de erro para vogais & 211 & & \\
\hline Matriz de Confusão & & & \\
Acerto de consoantes & 114 & 240 & $48 \%$ \\
Substituição de consoantes & 99 & 240 & $41 \%$ \\
Omissões de consoantes & 27 & 240 & $11 \%$ \\
\hline
\end{tabular}




\section{Caso An}

O desempenho de An está descrito na Tabela 2 e observase que, na aplicação da lista de palavra, foram verificados $47 \%$ de acerto de palavras, $88 \%$ de acerto de fonemas, $87 \%$ de padrão total de erros de fonemas, $83 \%$ de padrão total de erro de consoantes e $92 \%$ de padrão total de erro de vogais. À Matriz de Confusão (Anexo 3), houve $73 \%$ de acerto de consoantes, $19 \%$ de substituições e $8 \%$ de omissões de consoantes.

Tabela 2. Descrição do desempenho de An na tarefa de reconhecimento de palavras

\begin{tabular}{lccc}
\hline & $\mathrm{N}$ & Total & $\%$ \\
\hline WASP & & & \\
Acerto de palavras & 45 & 96 & $47 \%$ \\
Acerto de fonemas & 416 & 474 & $88 \%$ \\
Padrão total de erro de fonemas & 843 & 967 & $87 \%$ \\
Padrão total de erro para consoantes & 605 & 732 & $83 \%$ \\
Padrão total de erro para vogais & 216 & 235 & $92 \%$ \\
\hline Matriz de Confusão & & & \\
Acerto de consoantes & 174 & 240 & $73 \%$ \\
Substituição de consoantes & 46 & 240 & $19 \%$ \\
Omissões de consoantes & 20 & 240 & $8 \%$ \\
\hline
\end{tabular}

\section{Caso Gui}

O desempenho de Gui está descrito na Tabela 3 e observa-se que, na aplicação da lista de palavra, foram verificados $86 \%$ de acerto de palavras, $95 \%$ de acerto de fonemas, $96 \%$ de padrão total de erros de fonemas, $94 \%$ de padrão total de erro de consoantes e $98 \%$ de padrão total de erro de vogais. À Matriz de Confusão (Anexo 4), houve $92 \%$ de acerto de consoantes, $7 \%$ de substituições e $1 \%$ de omissões de consoantes.

Tabela 3. Descrição do desempenho de Gui na tarefa de reconhecimento de palavras

\begin{tabular}{lccc}
\hline & $\mathrm{N}$ & Total & $\%$ \\
\hline WASP & & & \\
Acerto de palavras & 83 & 96 & $86 \%$ \\
Acerto de fonemas & 379 & 400 & $95 \%$ \\
Padrão total de erro de fonemas & 746 & 780 & $96 \%$ \\
Padrão total de erro para consoantes & 534 & 571 & $94 \%$ \\
Padrão total de erro para vogais & 205 & 209 & $98 \%$ \\
\hline Matriz de Confusão & & & \\
Acerto de consoantes & 174 & 190 & $92 \%$ \\
Substituição de consoantes & 15 & 190 & $7 \%$ \\
Omissões de consoantes & 1 & 190 & $1 \%$ \\
\hline
\end{tabular}

\section{Caso Re}

O desempenho de Re está descrito na Tabela 4 e observase ocorrência de $10 \%$ de acerto de palavras, $56 \%$ de acerto de fonemas, $57 \%$ de padrão total de erros de fonemas, $34 \%$ de padrão total de erro de consoantes e $76 \%$ de padrão total de erro de vogais. À Matriz de Confusão (Anexo 5), houve $37 \%$ de acerto de consoantes, $38 \%$ de substituições e $25 \%$ de omissões de consoantes.

Tabela 4. Descrição do desempenho de Re na tarefa de reconhecimento de palavras

\begin{tabular}{lccc}
\hline & N & Total & $\%$ \\
\hline WASP & 5 & 48 & $10 \%$ \\
Acerto de palavras & 134 & 240 & $56 \%$ \\
Acerto de fonemas & 273 & 480 & $57 \%$ \\
Padrão total de erro de fonemas & 124 & 360 & $34 \%$ \\
Padrão total de erro para consoantes & 91 & 120 & $76 \%$ \\
Padrão total de erro para vogais & 91 & & \\
\hline Matriz de Confusão & & 120 & $37 \%$ \\
Acerto de consoantes & 44 & 120 & $38 \%$ \\
Substituição de consoantes & 46 & 120 & $25 \%$ \\
Omissões de consoantes & 30 & & \\
\hline
\end{tabular}

\section{DISCUSSÃO}

$\mathrm{Na}$ análise do desempenho de Ka no reconhecimento de palavras, a percentagem de palavras corretas, em geral utilizadas na caracterização desta habilidade, é o que menos fornece dados sobre sua habilidade de percepção de sons da fala. Ka acertou somente $27 \%$ das palavras apresentadas, no entanto, quando consideramos a percentagem de acerto de fonemas $(68 \%)$, a percentagem de traços corretos nas consoantes $(56 \%)$ e a percentagem de vogais corretas $(85 \%)$, fica evidente que Ka tem dificuldades específicas no reconhecimento de consoantes. Uma análise do comportamento da criança, sobre o traço mais freqüentemente confundido por ela, revela que o ponto de articulação foi, de modo geral, o traço que mais comprometeu o reconhecimento, aspecto facilmente sinalizado tanto na Matriz de Confusão (Anexo 2), quanto nas tabelas geradas pelo WASP.

A diferença entre o acerto de vogal e de consoante observado na Tabela 1 se dá pelo fato de que as vogais são mais facilmente detectadas auditivamente e, assim, mascaram as consoantes que tendem a serem substituídas ou omitidas. $\mathrm{O}$ estabelecimento de decisões e metas terapêuticas, quanto ao trabalho auditivo, dependeu da análise da audibilidade deste traço para Ka, que determinou estratégias auditivas ou visuais para melhor reconhecimento de palavras. Sendo assim, as metas traçadas foram no sentido de trabalhar com seu potencial auditivo, já que Ka não demonstrou estar utilizando este potencial no reconhecimento de palavras. Também foi proposta a revisão dos AASIs e o estabelecimento de metas terapêuticas que levassem a melhor desempenho na percepção de fala.

Durante a análise das produções de An, por meio das listas de palavras e da Matriz de Confusão (Anexo 3), foi possível observar que ele apresenta uma boa produção de fala se relacionada ao grau de sua perda auditiva. Avaliou-se que a porcentagem do acerto de fonemas é praticamente o dobro 
da porcentagem do acerto de palavras e as substituições fonêmicas encontram-se na região dos fonemas fricativos. A diferença entre o acerto de vogal e de consoante visualizado na Tabela 2 se deu pelo fato de que as vogais são mais facilmente detectadas auditivamente e, assim, mascaram as consoantes que tendem a serem substituídas ou omitidas. Este desempenho parece indicar que modificações nas características acústicas dos AASI podem ser necessárias, visando enfatizar freqüências mais agudas e, conseqüentemente, facilitar a percepção de fonemas fricativos.

Ao analisar as produções de Gui, observou-se que a porcentagem de palavras é pior do que a de fonemas e, além disso, a porcentagem de fonemas é mais próxima do padrão total de erros. $\mathrm{O}$ valor da porcentagem de padrão de erro das consoantes é próximo da porcentagem de acerto das consoantes (Tabela 3). No caso Gui, sujeito com bom uso da audição residual, as diferenças entre os tipos de medida são pequenas, isto é, há pouco espaço para melhora, pois ele já discriminava bem os sons da fala (Anexo 4), o que remete a um bom prognóstico para o uso do AASI. O trabalho terapêutico neste caso enfoca situações de comunicação de grupo, ou na presença de ruído e as estratégias compensatórias.

$\mathrm{Na}$ análise das produções de Re, observou-se que há pouco aproveitamento auditivo deste sujeito. Tal fato relacionase com a demora para a família procurar indicação de $\mathrm{AASI}^{(29)}$. Uma grande diferença observada foi em relação ao acerto de palavras e de fonemas, em que a produção de Re apresentou uma porcentagem pequena de acerto de palavras (10\%), embora seja possível notar grande discrepância em relação à contagem de fonemas (56\%). A diferença entre o acerto de vogal e de consoante observada na Tabela 4 se dá pelo fato de que as vogais são mais facilmente detectadas auditivamente e, assim, mascaram as consoantes que tendem a serem substituídas ou omitidas. Desta forma, a porcentagem do acerto de consoantes dá a direção do potencial auditivo desta criança e pode ser utilizada como diretriz da intervenção a ser desenvolvida na terapia, a fim de que seja feito um investimento de trabalho auditivo voltado para potencializar percepção auditiva para o ponto de articulação (Anexo 5), o qual apresentou maior quantidade de erros. Assim, Re pode se beneficiar de um melhor aproveitamento auditivo.

Os critérios decorrentes da proposta do WASP foram os mais sensíveis para as tentativas de acerto produzidas pelo sujeito, que não resultaram em acerto de palavra propriamente dito do potencial auditivo do sujeito. Os critérios da Matriz de Confusão permitiram melhor especificação dos erros quanto a substituições e omissões ${ }^{(20)}$. Com relação à não considerar o arquifonema /R/ nos verbos no infinito, verificamos que embora no português falado esse fonema não seja comumente utilizado, não sendo, portanto, considerado como um erro na análise das produções das crianças, sugerimos que essas palavras não sejam escolhidas pelos próximos grupos que realizarem esse instrumento de avaliação de percepção e produção de fala, uma vez que não se configura como erro para a contagem das fonêmica, portanto, não se trata de dado significativo para análise.

A análise de diferentes critérios a partir da adaptação do WASP e da Matriz de Confusão permitiu um maior detalhamento das habilidades de percepção e produção de fala de cada sujeito. Apenas a utilização da percentagem de acertos de palavras subestima o potencial de Ka e Gui e não fornece diretrizes quanto às decisões quanto à amplificação e indicação terapêutica. Desta forma, a combinação dos critérios decorrentes de duas propostas contribui para um melhor entendimento das características de percepção e produção de fala de cada sujeito.

Os dados corroboram para o fato de que a utilização conjunta dos critérios estudados permite encontrar intervenções e estratégias terapêuticas de modo a oferecer mudanças qualitativas no reconhecimento das pistas acústicas da fala, modificando a produção da fala do deficiente auditivo ${ }^{(8,12,20,24,26)}$.

As implicações deste tipo de análise são importantes para a prática clínica no trabalho fonoaudiológico com sujeitos com perda auditiva, pois permitem verificar a relação entre as diferentes características acústicas de sistemas de amplificação e o desenvolvimento dos sujeitos na terapia com ênfase na percepção auditiva. Por meio da comparação dos resultados, em dois momentos distintos de avaliação da produção e percepção de fala, foi possível observar a ocorrência de mudanças no tipo de erro apresentado pelo paciente ${ }^{(20,26)}$, o que pode direcionar o estabelecimento de estratégias terapêuticas que contemplassem as possibilidades do sujeito. Este estudo aponta para a necessidade de desenvolvimento de novas pesquisas nessa temática, ampliando a utilização de critérios qualitativos na interpretação dos erros em tarefas de reconhecimento de palavras.

\section{CONCLUSÃO}

A partir da necessidade de critérios de avaliação do progresso da criança com deficiência auditiva no processo terapêutico, quanto à linguagem, audição e oralidade, foi possível analisar e discutir a avaliação da percepção de fala de crianças com deficiência auditiva usuárias de AASI através de critérios qualitativos na análise de erros em tarefa de reconhecimento de palavras. As relações entre os diferentes critérios de análise permitiram ampliar a compreensão dos fatores diretamente relacionados ao desempenho das crianças no reconhecimento de palavras. Possibilitou também uma análise mais detalhada do potencial auditivo com implicações importantes para o processo terapêutico com ênfase na audição residual. Ao combinar diferentes critérios de análise dos erros é possível ampliar o conhecimento das habilidades de percepção de fala dos sujeitos e, conseqüentemente, contribuir para o estabelecimento de metas terapêuticas adequadas às suas necessidades. 


\begin{abstract}
Purpose: To compare different error analysis criteria in words recognition tasks, in order to determine its potential for clinical utilization with hearing impaired children, particularly in the relations between speech perception and residual hearing. Methods: Lists of words were presented to four hearing impaired children, with ages varying from six to eight years, who used hearing aids. The results were analyzed using different criteria, based on two different instruments: WASP: percentage of correct words, phonemes and total error pattern; Confusion Matrix: substitutions and omission of phonemes. Results: The analysis including criteria of both instruments allowed for considerations on use of residual hearing and the influence of intervention on each child's performance. Conclusion: The combination of different criteria for error analysis allows greater knowledge about speech perception abilities of the subjects and, consequently, provides greater specificity in clinical techniques in aural rehabilitation.
\end{abstract}

Keywords: Hearing loss; Auditory perception; Hearing aids/utilization

\title{
REFERÊNCIAS
}

1. Silman S, Iório MCM, Mizhahi MM, Parra VM. Próteses auditivas: um estudo sobre seu benefício na qualidade de vida de indivíduos portadores de perda auditiva neurossensorial. Distúrb Comun. 2004;16(2):153-65.

2. Kaminski JM, Tochetto TM, Mota HB. Maturação da função auditiva e desenvolvimento de linguagem. Rev Soc Bras Fonoaudiol. 2006;11(1):17-21.

3. Simonetti P, Costa Filho OA. Seleção e adaptação de AASI para bebês: verificação in situ das diferentes caracteristicas morfofisiológicas da orelha externa. Distúrb Comun. 2003;15(2):201-21.

4. Ferreira MCM. E quando o cliente é o bebê? Particularidades da intervenção fonoaudiólogica em bebês portadores de deficiência auditiva. Distúrb Comun. 2003;15(2):355-82.

5. Balieiro CR, Ficker LB. Reabilitação aural: a clínica fonoaudiológica e o deficiente auditivo. In: Lopes Filho OC, editor. Tratado de fonoaudiologia. São Paulo: Roca; c1997. p. 311-325.

6. Nery DM, Novaes B. Identificação de estratégias no processo terapêutico de uma criança deficiente auditiva. Distúrb Comun. 2001;13(1):49-67.

7. Chelucci LSO, NOVAES BC. Contar histórias com livros infantis: caracterização da interação das díades mãe ouvinte/criança com deficiência auditiva e mãe/criança ouvintes. Distúrb Comun. 2005;17(1):55-67.

8. Melo ME, Novaes BCAC. Caderno de experiências no processo terapêutico de uma criança portadora de deficiência auditiva. Pró-Fono. 2001;13(2):242-8.

9. Soares AD, Chiari BM. Caracterização da narrativa oral de deficientes auditivos. Rev Soc Bras Fonoaudiol. 2006;11(4):272-8.

10. Novaes BC, Balieiro CR. Terapia fonoaudiológica da criança surda. In: Ferreira LP, Befi-Lopes DM, Limongi SCO, organizadores. Tratado de Fonoaudiologia. São Paulo: Roca; 2004.

11. Caporali SA, Arieta A. Reconhecimento de fala no ruído e estudo comparativo entre grupos com e sem queixa de percepção de fala. Rev Soc Bras Fonoaudiol. 2004;9(3):129-35.

12. Granha DO. A utilização de músicas infantis na terapia fonoaudiológica da criança deficiente auditiva. Distúrb Comun. 2002;14(1):188-9.

13. Dreossi RCF, Momensohn-Santos TM. Investigação sobre o efeito do ruído na inteligibilidade de fala de crianças da quarta série do ensino fundamental. Rev Soc Bras Fonoaudiol. 2004;9(3):136-43.

14. Geers AE. Techniques for assessing auditory speech perception and lipreading enhancement in young deaf children. Volta Rev. 1994;96(5):8596.
15. Orlandi ACL. Procedimento de avaliação de percepção da fala em deficiência auditiva profunda nos primeiros anos de vida [tese]. São Paulo: Pontifícia Universidade Católica de São Paulo; 1996.

16. Delgado EMC. Elaboração de um procedimento de avaliação da percepção dos sons da fala para crianças deficientes auditivas [tese]. São Paulo: Pontifícia Universidade Católica de São Paulo; 1997.

17. Valente SLOL. Elaboração de listas de sentenças construídas na língua portuguesa [tese]. São Paulo: Pontifícia Universidade Católica de São Paulo; 1998.

18. Frederigue NB. Otimização da percepção da fala em deficientes auditivos usuários do sistema de implante coclear multicanal [tese]. São Paulo: Pontifícia Universidade Católica de São Paulo; 2000.

19. Buzo BC, Ubrig MT, Novaes BC. Adpatação de aparelho de amplificação sonora individual: relações entre a auto-percepção do handicap auditivo e a avaliação da percepção de fala. Distúrb Comun. 2004;6(1):17-25.

20. Helou LF, Novaes BC. Utilização da matriz de confusão na indicação de aparelho de amplificação sonora individual. Distúrb Comun. 2005;17(2):203-13.

21. Wertzner HF, Papp ACCS, Amaro L, Galea DES. Relação entre os processos fonológicos e classificação perceptiva de inteligibilidade de fala no transtorno fonológico. Rev Soc Bras Fonoaudiol. 2005;10(4):193-200.

22. Santos FA, Spinelli M. A discriminação auditiva e o desempenho escolar. Distúrb Comun. 2003;14(2):309-18.

23. Miller G, Nicely P. An analysis of perceptual confusion among some English consonants. J Acoust Soc Am. 1955;27:338-53.

24. Araújo AMLA. Percepção de fala residual [tese]. Campinas: Universidade Estadual de Campinas; 1998.

25. Koch ME. Bringing sounds to life: principles and practices of cochlear implant rehabilitation. Timonium (MD): York Press; 1999.

26. Padilha RB. Percepção de fala: parâmetros de desempenho e implicações na intervenção fonoaudiológica com crianças deficientes auditivas. Distúrb Comun. 2004;16(3):414-5.

27. Harris RW, Goffi MVS, Pedalini MEB, Merrill A, Gygi MA. Reconhecimento de palavras dissilábicas psicometricamente equivalentes no Português Brasileiro faladas por indivíduos do sexo masculino e do sexo feminino. Pró-Fono. 2001;13(2):249-62.

28. Bagno M. Preconceito lingüístico: o que é, como se faz. 2a ed. São Paulo: Loyola; 1999.

29. Moeller MP. Early intervention and language development in children who are deaf and hard of hearing. Pediatrics. 2000;106(3):E43. 
Anexo 1. Exemplo de lista de palavra usada em WASP para elaboração de cada terapeuta segundo a familiridade lingüística de cada paciente

Lista 1 - Padrăo total de erro - Novaes et al (2003).

Nome:

Examinador:

Data: _L_

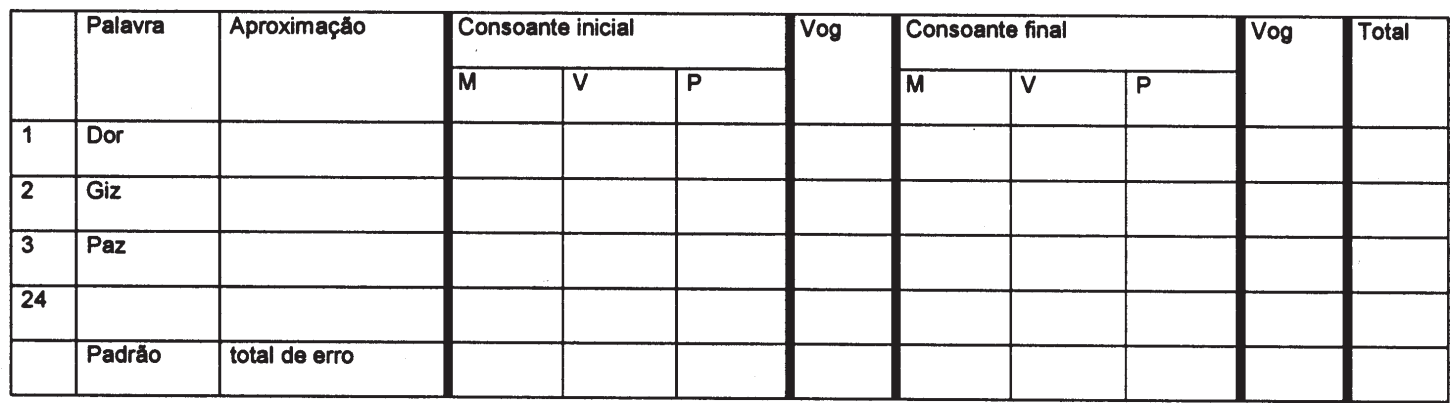

Anexo 2. Matriz de Confusão do sujeito Ka.

\begin{tabular}{|c|c|c|c|c|c|c|c|c|c|c|c|c|c|c|c|c|c|c|c|c|c|c|c|c|}
\hline \multicolumn{25}{|c|}{ MATRIZ DE CONFUSÃO* } \\
\hline Ka & -10 & $1 / 07 / 0$ & & & & & & & & & & & & & & & & & & & & & & \\
\hline$E E^{R}$ & $|\mathrm{p}|$ & $|t|$ & $\mid \mathrm{k} /$ & $\mid \mathrm{b} /$ & $/ \mathrm{d} /$ & $|g|$ & |fl & $|\mathrm{s}|$ & $15 /$ & $\mid t 5 /$ & $|\mathrm{v}|$ & $|z|$ & |3/ & $\mid \mathrm{d} /$ & $\mid \mathrm{m} /$ & $\ln /$ & $|\mathrm{n}|$ & /1/ & $|\mathrm{A}|$ & $|\mathrm{r}|$ & $|\mathrm{R}|$ & (3) & & total \\
\hline $\mid \mathrm{p} /$ & 17 & 2 & & 6 & & & & & & & & & & & & & & & & & & 1 & $\mid \mathrm{p} /$ & 26 \\
\hline$|t|$ & 2 & 8 & 3 & & 3 & 1 & & & & & & & & & & & & & & & & 1 & $|t|$ & 18 \\
\hline$/ \mathrm{k} /$ & & 3 & 12 & 1 & 2 & 1 & & & & & & & & & & & & & & & & & $/ \mathrm{k} /$ & 19 \\
\hline$/ \mathrm{b} /$ & 2 & & & $\overline{10}$ & 1 & & & & & & & & & & & & & & & & & & $/ \mathrm{b} /$ & 13 \\
\hline$/ \mathrm{d} /$ & & 1 & 1 & 4 & 2 & & & & & & 1 & & & & & & & & & & & 1 & /d/ & 10 \\
\hline $\mid \mathrm{g} /$ & & & & 2 & 1 & & & & & & 1 & & & & & & & & & & & 1 & $\mid g /$ & 5 \\
\hline |f/ & & 2 & & 3 & & & 5 & & & & & & & & & & & & & & & & |f/ & 10 \\
\hline$|s|$ & 3 & 8 & 1 & 1 & & & & 2 & & & & & & & & & & 1 & & & & 6 & $|\mathrm{~s}|$ & 22 \\
\hline $15 /$ & & & & & & & & & 5 & & 1 & & & & & & & & & & & & 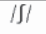 & 6 \\
\hline$/ \mathrm{t} /$ & & & & & & & & & & & & & & & & & & & & & & & $/ \mathrm{t} /$ & 0 \\
\hline$|\mathrm{v}|$ & & & & 1 & 1 & 1 & & & & & 8 & & & & 2 & & & & 1 & & & & $|\mathrm{v}|$ & 14 \\
\hline$|z|$ & & 1 & & 2 & 1 & & & & & & & & & & & & & & & & & & $|z|$ & 4 \\
\hline$|3|$ & & & & & & & & & & & & & 4 & & & & & & & & & 1 & |3/ & 5 \\
\hline $\mid \mathrm{d} /$ & & & & & & & & & & & & & & & & & & & & & & & $/ \mathrm{d} / /$ & 0 \\
\hline$/ \mathrm{m} /$ & 1 & & 1 & 8 & 1 & & & & & & & & & & 6 & & & & & & & 2 & $\mathrm{Im} /$ & 19 \\
\hline$/ \mathrm{n} /$ & & 2 & & 2 & 2 & & & & & & 1 & & & & 1 & 4 & & 1 & & & & 2 & $\mid \mathrm{n} /$ & 14 \\
\hline$/ \mathrm{s} /$ & & 1 & & & & 1 & & & & & & & & & & & 3 & & & & & 2 & $/ \mathrm{p} /$ & 7 \\
\hline 111 & & & 1 & & & & & & & & 1 & & & & & & & 10 & & & & 5 & /11 & 17 \\
\hline$|N|$ & & & & & & & & & & & & & & & & & & 3 & 1 & & & 2 & $|\mathrm{~N}|$ & 7 \\
\hline $\mid \mathrm{r} /$ & & & & & 1 & & & & & & & & & & & & & & & 16 & & 3 & $|\mathrm{r}|$ & 20 \\
\hline $\mid \mathrm{R} /$ & & & & 1 & 1 & & & & & & & & & & & & & 1 & & & 1 & & $\mid R /$ & 4 \\
\hline & & Total & e apre & ntaçô & & 240 & $100 \%$ & & & & & & & & & & & & & & & & & \\
\hline & & Núme & de a & & & 114 & $48 \%$ & & & & & & & & & & & & & $E=E s$ & mulo & & & \\
\hline & & Núme & de or & issões & & 27 & $11 \%$ & & & & & & & & & & & & & $R=R$ & posta & & & \\
\hline & & Númr & de su & tituiç & & 99 & $41 \%$ & & & & & & & & & & & & & & & & & \\
\hline
\end{tabular}


Anexo 3. Matriz de Confusão do sujeito An.

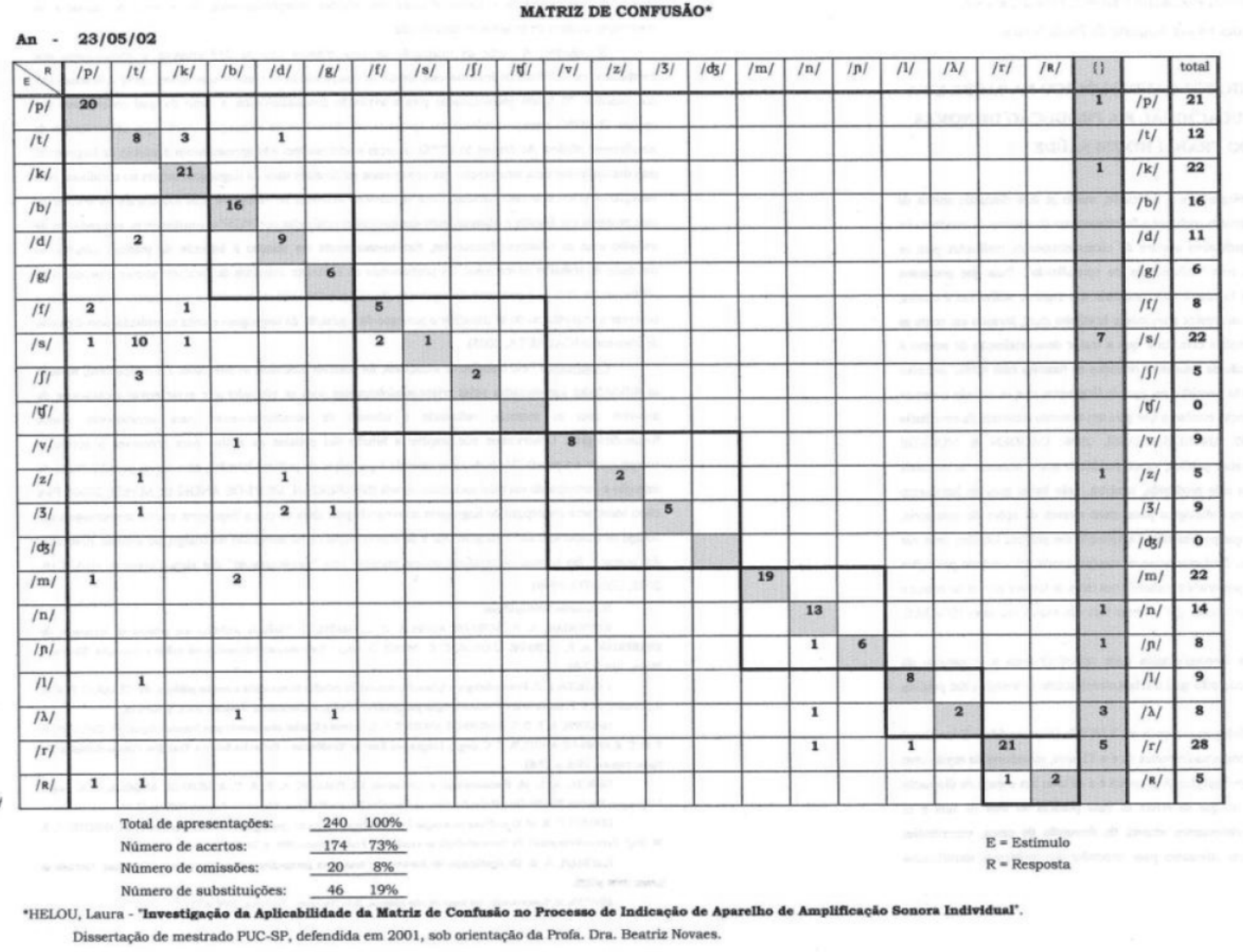


Anexo 4. Matriz de Confusão do sujeito Gui.

MATRIZ DE CONFUSÃO*

\section{Gui $-26 / 10 / 02$}

\begin{tabular}{|c|c|c|c|c|c|c|c|c|c|c|c|c|c|c|c|c|c|c|c|c|c|c|c|c|}
\hline$E^{R}$ & $\mid \mathrm{p} /$ & $|t|$ & $/ \mathrm{k} /$ & $/ \mathrm{b} /$ & $/ \mathrm{d} /$ & $\mid \mathrm{g} /$ & /f/ & $|\mathrm{s}|$ & $\mathrm{IJ/}$ & $\mid 15 /$ & $\mid \mathrm{v} /$ & $|z|$ & $|3|$ & $\left|\mathrm{d}_{3}\right|$ & $\mid \mathrm{m} /$ & $\ln /$ & $/ \mathrm{s} /$ & /1/ & $/ \mathrm{M} /$ & $\mid \mathrm{r} /$ & $\mid \mathrm{R} /$ & \{\} & & total \\
\hline $\mid \mathrm{p} /$ & 14 & 2 & & & & & 2 & & & & & & & & & & & & & & & & $|\mathrm{p}|$ & 18 \\
\hline$|t|$ & 1 & 14 & & & & & & & & & & & & & & & & & & & & & $|t|$ & 15 \\
\hline $\mid \mathrm{k} /$ & & 1 & 16 & & & & & & & & & & & & & & & & & & & & $/ \mathrm{k} /$ & 17 \\
\hline$/ \mathrm{b} /$ & & & & 10 & & & & & & & & & & & & & & & & & & & $/ \mathrm{b} /$ & 10 \\
\hline$/ d /$ & & & & & 10 & & & & & & & & & & & & & & & & & & $/ \mathrm{d} /$ & 10 \\
\hline$|\mathrm{g}|$ & & & & & & 3 & & & & & 1 & & & & & & & & & & & & $|g|$ & 4 \\
\hline /f/ & 2 & & & & & & 7 & & & & & & & & & & & & & & & 1 & $\mid \mathrm{f} /$ & 10 \\
\hline $\mid \mathrm{s} /$ & 1 & 1 & & & & 1 & & 11 & & & & & & & & & & & & & & & $\mid \mathrm{s} /$ & 14 \\
\hline$/ 5 /$ & & & & & & & & & 5 & & & & & & & & & & & & & & $/ \mathrm{d} /$ & 5 \\
\hline$/ 5 /$ & & & & & & & & & & & & & & & & & & & & & & & 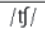 & 0 \\
\hline $\mid \mathrm{v} /$ & & & & & & & & & & & 11 & & & & & & & & & & & & $/ \mathrm{v} /$ & 11 \\
\hline$|z|$ & & & & & & & & & & & & 3 & & & & & & & & & & & $|z|$ & 3 \\
\hline $13 /$ & & & & & & & & & & & 1 & & 3 & & & & & & & & & & $13 /$ & 4 \\
\hline $\mid \mathrm{d} /$ & & & & & & & & & & & & & & & & & & & & & & & $\mid \mathrm{d} /$ & 0 \\
\hline$/ \mathrm{m} /$ & & & & & & & & & & & & & & & 16 & & & & & & & & $\mid \mathrm{m} /$ & 16 \\
\hline $\mathrm{ln} /$ & & & & & & & & & & & & & & & & 13 & & & & & & & $\mid \mathrm{n} /$ & 13 \\
\hline$/ \mathrm{n} /$ & & & & & & & & & & & & & & & & & 7 & & & & & & $/ \mathrm{n} /$ & 7 \\
\hline /11 & & & & & & & & & & & & & & & & & & 13 & & & & & /1/ & 13 \\
\hline$|A|$ & & & & & & & & & & & & & & & & & & & 5 & & & & $\mid \mathrm{N} /$ & 5 \\
\hline$|\mathrm{r}|$ & & & & & & & & & & & & & & & & & & & & 10 & & & $\mid \mathrm{T} /$ & 10 \\
\hline$/ \mathrm{R} /$ & & & & & & & & & & & & & & & 1 & & & 1 & & & 3 & & $\mid \mathrm{R} /$ & 5 \\
\hline & & \multicolumn{4}{|c|}{ Total de apresentaçôes: } & 190 & $100 \%$ & & & & & & & & & & & & & & & & & \\
\hline & & \multicolumn{3}{|c|}{ Número de acertos: } & & 174 & $92 \%$ & & & & & & & & & & & & & \multicolumn{2}{|c|}{$\mathrm{E}=\mathrm{Estimulo}$} & & & \\
\hline & & \multicolumn{4}{|c|}{ Número de omissões: } & 1 & $1 \%$ & & & & & & & & & & & & & \multicolumn{2}{|c|}{$R=$ Resposta } & & & \\
\hline & & \multicolumn{4}{|c|}{ Nủmero de substituições: } & 15 & $7 \%$ & & & & & & & & & & & & & & & & & \\
\hline
\end{tabular}

•HEL.OU, Laura - 'Investigaçăo da Aplicabilidade da Matriz de Confusăo no Processo de Indicaạăo de Apareltho de Amplificaạăo Sonora Individual". 
Anexo 5. Matriz de Confusão do sujeito Re.

MATRIZ DE CONFUSÃO*

\section{$\operatorname{Re}-23 / 08 / 02$}

\begin{tabular}{|c|c|c|c|c|c|c|c|c|c|c|c|c|c|c|c|c|c|c|c|c|c|c|c|c|}
\hline$E V^{R}$ & $|\mathrm{p}|$ & $|t|$ & $|\mathrm{k}|$ & $/ \mathrm{b} /$ & $/ \mathrm{d} /$ & $|g|$ & $|\mathrm{f}|$ & $|\mathrm{s}|$ & $\mid \mathrm{J} /$ & $|\mathrm{t}|$ & $|\mathrm{v}|$ & $|z|$ & $|3|$ & $\left|\mathrm{d}_{3}\right|$ & $/ \mathrm{m} /$ & $\mid \mathrm{n} /$ & $/ \mathrm{n} /$ & /1] & $|\mathrm{N}|$ & $\mid \mathrm{r} /$ & $|\mathrm{R}|$ & \{\} & & total \\
\hline$|\mathrm{p}|$ & 3 & 3 & & 1 & & & 1 & & & & & & & & 2 & & & & & & & 1 & $\mid \mathrm{p} /$ & 11 \\
\hline$|t|$ & 1 & 5 & & & & & 1 & & & & & & & & & & & & & & & 3 & $|t|$ & 10 \\
\hline $\mid \mathrm{k} /$ & 1 & 1 & 2 & 1 & & & 1 & & & & 1 & & & & & 1 & & & & & & 5 & $|\mathrm{k}|$ & 13 \\
\hline$/ \mathrm{b} /$ & & & & 6 & & & & & & & & & & & & & & & & & & 1 & $/ \mathrm{b} /$ & 7 \\
\hline$/ \mathrm{d} /$ & & & & & 3 & & 1 & & & & 1 & & & & & & & & & & & 1 & $\mid \mathrm{d} /$ & 6 \\
\hline $\mid \mathrm{g} /$ & 1 & 1 & & & 1 & & & & & & & & & & & & & & & & & 1 & $\mid \mathrm{g} /$ & 4 \\
\hline$|\mathrm{f}|$ & 2 & 1 & & & & & 1 & & & & & & & & & 1 & & & & & & & $|\mathrm{f}|$ & 5 \\
\hline$|\mathrm{s}|$ & & 3 & & & & & & 1 & & & & & & & & & & & & & & 1 & $\mid \mathrm{s} /$ & 5 \\
\hline 151 & & & & & & & & & 4 & & & & & & & & & & & & & & $\mid \mathrm{S} /$ & 4 \\
\hline $\mid \mathrm{t} /$ & & & & & & & & & & & & & & & & & & & & & & & $|t|$ & 0 \\
\hline$|\mathrm{v}|$ & & 1 & & & & & & & & & 2 & & & & & & & 1 & & & & 4 & $|\mathrm{v}|$ & 8 \\
\hline$|z|$ & & & & & & & & & & & 1 & 1 & & & & & & & & & & & $|z|$ & 3 \\
\hline 131 & & & & & & & & & 1 & & & & 1 & & & & & & & & & 1 & $|3|$ & 3 \\
\hline$\left|\mathrm{d}_{3}\right|$ & & & & & & & & & & & & & & & & & & & & & & & $\left|\mathrm{d}_{3}\right|$ & 0 \\
\hline$/ \mathrm{m} /$ & & & & 1 & & & & 1 & & & & & & & 3 & & & 1 & & & & 1 & $\mid \mathrm{m} /$ & 7 \\
\hline $\mid \mathrm{n} /$ & 1 & 1 & & & & & & & & & 1 & & & & 1 & 4 & & 1 & & & & & $\mid \mathrm{n} /$ & 9 \\
\hline $\mid \mathrm{n} /$ & & & & & & & & & & & & & & & & & & 1 & 1 & & & 4 & $/ \mathrm{n} /$ & 7 \\
\hline /1/ & & & & 1 & & & & & & & 1 & & & & & & & 4 & & & & 5 & $/ 11$ & 11 \\
\hline$|\mathrm{N}|$ & & & & & & & & & & & & & & & & & & 1 & & & & 2 & $|\lambda|$ & 3 \\
\hline$|\mathrm{r}|$ & & & & & & & & & & & & & & & & & & & & 3 & & 1 & $\mid \mathrm{r} /$ & 4 \\
\hline $\mid \mathrm{R} /$ & & & & & & & & & & & 1 & & & & & & & & & & 1 & 1 & $/ \mathrm{R} /$ & 3 \\
\hline
\end{tabular}

\begin{tabular}{l|l|l} 
Total de apresentações: & $120 \quad 100 \%$ \\
\hline
\end{tabular}

\begin{tabular}{l|l|l|}
\hline Número de acertos: & 44 & $37 \%$ \\
\hline
\end{tabular}

$\mathrm{E}=$ Estimulo

\begin{tabular}{l|l|l|} 
Número de omissôes: & 30 & $25 \%$ \\
\hline
\end{tabular}

$R=$ Resposta

\begin{tabular}{l|l|l} 
Número de substituições: & 46 & $38 \%$ \\
\hline
\end{tabular}

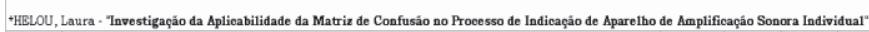

Disserta̧̧ão de mestrado PUC-SP, defendida em 2001, sob orientą̧ẫo da Profa. Dra. Beatriz Novaeg. 